\title{
Influence of viscous fingering on dynamic saturation- pressure curves in porous media
}

\author{
G. Løvoll ${ }^{1,5}$ (grunde.lovoll@fys.uio.no), M. Jankov ${ }^{1}$, K. J. \\ Måløy ${ }^{1}$, R. Toussaint ${ }^{2}$, J. Schmittbuhl ${ }^{2}$, G. Schäfer ${ }^{3}$ and Y. \\ Méheust ${ }^{4}$ \\ ${ }^{1}$ Department of Physics, University of Oslo, P.O. Box 1048 Blindern, NO-0316, \\ Oslo, Norway \\ ${ }^{2}$ Institut de Physique du Globe de Strasbourg, CNRS, Université Louis Pasteur, 5
rue Descartes, 67084 Strasbourg Cedex, France
}

${ }^{3}$ Laboratoire d'Hydrologie et de Géochimie de Strasbourg, 1 rue Blessig, 67083 Strasbourg Cedex, UMR 7517 CNRS - Université de Strasbourg, France

${ }^{4}$ Geosciences Renne (UMR CNRS 6118), Université Rennes 1, Rennes, France

${ }^{5}$ Det Norske Veritas, Research and Innovation, Veritasveien 1, NO-1322 Høvik, Norway

Abstract. We report on results from primary drainage experiments on quasi-twodimensional porous models. The models are transparent, allowing the displacement process and structure to be monitored in space and time during water retention experiments carried out at various speeds. By combining detailed information on the displacement structure with global measurements of pressure, saturation and the capillary number $\mathrm{Ca}$, we obtain a scaling relation relating pressure, saturation, system size and capillary number. This scaling relation allows pressure-saturation curves for a wide range of capillary numbers to be collapsed on the same master curve.

Keywords: drainage, dynamic capillary pressure, saturation, water retention, viscous effects, rescaling

\section{Introduction}

Different types of fluid displacements in porous media play important roles in many natural and commercial processes (Bear, 1972; Dullien, 1992; Sahimi, 1995). Various multiphase problems have been studied and modeled over the last decades; see (Bear, 1972; Dullien, 1992; Sahimi, 1995; Lenormand, 1989) and references therein.

The morphology of the displacement structures observed in immiscible two phase flow, are in general controlled by the competition between

(C) 2010 Kluwer Academic Publishers. Printed in the Netherlands. 
viscous forces, gravitational forces and capillary forces; those various forces act on scales ranging from the pore scale to the system size. The relative wettabilities, viscosities, and densities of the fluids, as well as the heterogeneity of the underlying porous media, could all play important roles in the competition process (Lenormand, 1989; Birovljev et al., 1991; Måløy et al., 1985; Måløy et al., 1992; Frette et al., 1997).

To characterize two phase flow in porous media, it is common practice to define a set of dimensionless numbers that quantify fluid pair characteristics. One such number is the viscosity ratio $M=\mu_{\mathrm{nw}} / \mu_{\mathrm{w}}$, where $\mu_{\mathrm{nw}}$ is the dynamic viscosity of the non-wetting fluid and $\mu_{\mathrm{w}}$ is the dynamic viscosity of the wetting fluid. The relative magnitudes of viscous and capillary forces (on the pore scale) are quantified by the capillary number Ca:

$$
\mathrm{Ca}=\frac{\mu_{\mathrm{w}} v_{\mathrm{f}} a^{2}}{\gamma \kappa}
$$

where $v_{\mathrm{f}}$ is the Darcy velocity or specific discharge, $a$ is the characteristic pore size, $\gamma$ is the interface tension and $\kappa$ is the intrinsic permeability of the homogeneous and isotropic porous medium. The specific discharge, or filtration velocity, $v_{\mathrm{f}}$, is $v_{\mathrm{f}}=Q / A$ where $Q$ is the volumetric flow rate and $A$ is the cross section area perpendicular to the flow. In drainage, i.e. flow regimes where the non-wetting fluid replaces the wetting one, three limit flow regimes can be reached by tuning $M$ and $\mathrm{Ca}$ (Lenormand et al., 1983; Lenormand et al., 1988; Lenormand, 1989). If the flow rate is sufficiently low $(\mathrm{Ca} \ll 1)$, one reaches the capillary fingering regime (Lenormand et al., 1983; Lenormand and Zarcone, 1985), for which the displacement structure is controlled solely by the fluctuations in the capillary threshold pressures. This regime is shown to have strong analogies to invasion percolation (Lenormand and Zarcone, 1985; Chandler et al., 1982; Wilkinson and Willemsen, 1983), and the invasion structure is fractal (Mandelbrot, 1982; Feder, 1988 ) with a fractal dimension $D_{\mathrm{c}}=1.83 \pm 0.01$ (Lenormand and Zarcone, 1985; Lenormand and Zarcone, 1989). If the invasion rate is high, the displacement is either stable or unstable depending on the viscosity contrast $M$. If a fluid with high viscosity is invading a fluid with low viscosity $(M \geq 1)$, the resulting pressure field due to the viscous dominated displacement will act against the growth of the invasion front, leading to stabilization of the displacement front at a finite width (Saffman and Taylor, 1958; Lenormand et al., 1988; Lenormand, 1989; Frette et al., 1997). On the other hand, if the invading fluid is the less viscous one, the displacement is unstable and falls in the viscous fingering regime (Saffman and Taylor, 1958; Måløy et al., 1985). The invasion structure is fractal with a fractal dimension $D_{\mathrm{v}} \simeq$ 1.53 (Toussaint et al., 2005).

TiPM-Knut-Grunde_RT.tex; 14/06/2010; 9:06; p.2 
At intermediate capillary numbers, the visocus and capillary forces dominates on different length scales. At small scales capillary forces dominate the problem so that the invasion structure locally is that of capillary fingering, while viscous forces dominate the problem at larger scales, resulting in a morphology typical of viscous fingering at larger scales. The crossover length-scale between these two regimes $l_{\mathrm{c}}$ scales with the capillary number as (Løvoll et al., 2004; Toussaint et al., 2005):

$$
l_{\mathrm{c}} \propto \frac{a}{\mathrm{Ca}} .
$$

Above this crossover length scale the flow is destabilized by viscous forces and the displacement takes place in narrow branched channels (for $M<1$ ). Viscous fingering in disordered porous media is different from standard Saffman-Taylor fingering (Saffman and Taylor, 1958), obtained in empty straight channels (Hele-Shaw cells), where the fingers are compact and occupy $1 / 2$ of the system width $W$. In contrast, in disordered porous media these structures are branched, the invasion structure is fractal (Måløy et al., 1985) and it has been demonstrated that the invasion structure occupies a smaller fraction of the system $(0.4 \mathrm{~W})$ (Løvoll et al., 2004; Toussaint et al., 2005).

Many authors in the "physics community" have been mostly concerned with characterizing and understanding displacement patterns and local flow properties. Hydrogeologists and soil scientists, on the other hand, have studied such systems with the goal of finding empirical laws relating saturation and capillary pressure at the Darcy scale, that is, a meso-scale at which the medium and the flow are described by continuous mathematical fields. The basic laws of multiphase flows treated at mesoscopic scale as a continuum require a closure of partialflow Darcy relations. The key point of this closure is a functional relation between the capillary pressure (in a water-air system) and (water) saturation in the form of retention curves. The basic assumption underlying this continuous theory of multiphase flow is that for a given porous medium and a wetting-/nonwetting-liquid pair there is a well defined relation between capillary pressure and saturation as found from quasi-static measurements on representative volumes. The best known mathematical models to describe the relationship are those of (Leverett, 1941), (Brooks and Corey, 1964) and (Van Genuchten, 1980). In these models, the model parameters are fit parameter and they are functions of the characteristics of the pore space, such as the pore size distribution and the degree of connectivity of the network. As found in the early sixties, the retention curves may also depend on the direction and on the "displacement history" of the water-air front, further on referred as hysteresis effects (Poulavissilis, 1962; Mualem, 1976; Mualem, 
1984; Kool and Parker, 1987; Parker and Lenhard, 1987; Luckner and Van Genuchten, 1989; Lenhard et al., 1993; Stauffer and Kinzelbach, 2001). Based on the parameterization procedure proposed by (Parker et al., 1987), the known retention curves of a porous sample are often used to obtain capillary pressure-saturation curves for the different fluid pairs present in a multiphase system (e.g. (Helmig, 1997; Bohy et al., 2006)). Later studies have demonstrated that hysteresis effects are not the only additional influence on this relationship and they show that there are considerable dynamic effects on the measured capillary pressure curves (Bourgeat and Panfilov, 1998; Hassanizadeh and Gray, 1988; Hassanizadeh et al., 2002; Dahle et al., 2005). Based on the results of former experimental studies (Smiles et al., 1971; Stauffer, 1977), Hassanizadeh et al. (Hassanizadeh et al., 2002) suggested to account for dynamic effects on the retention curves by introducing a term of dynamic capillary pressure in the form of the sum of the capillary pressure obtained under hydrostatic equilibrium conditions, depending on the water saturation of the soil sample, and a dynamic pressure term expressed by a lumped parameter times the variation of water saturation over time.

The present paper addresses the gap between (i) capillary pressure as understood in the framework of a pore scale description and modeling of drainage, and (ii) capillary pressure as measured at the global scale of a sample or as described in Darcy-scale models. We present results from drainage experiments on synthetic quasi two-dimensional porous media, in which non-wetting air displaces a highly viscous wetting glycerolerin/water solution; hence, gravity has no influence on the displacement. We investigate the crossover regime between the regime of slow displacement (capillary fingering), and fast displacement (viscous fingering). By using transparent quasi two-dimensional porous media as our porous media, optical methods are used to observe and analyze the local and global displacement geometry and how it changes with capillary number. The combination of local and global information allow us to perform upscaling of our data from the pore scale to the scale of our experimental porous media. In this manner, we are able to relate pressure, saturation, capillary number and system size.

\section{Experimental setup}

The presented experiments are performed on quasi two-dimensional porous media. The thickness of the cells containing this porous medium is $a=1 \mathrm{~mm}$, and corresponds to the diameter of the fixed glass beads composing the solid matrix of this porous medium. The porous medium 


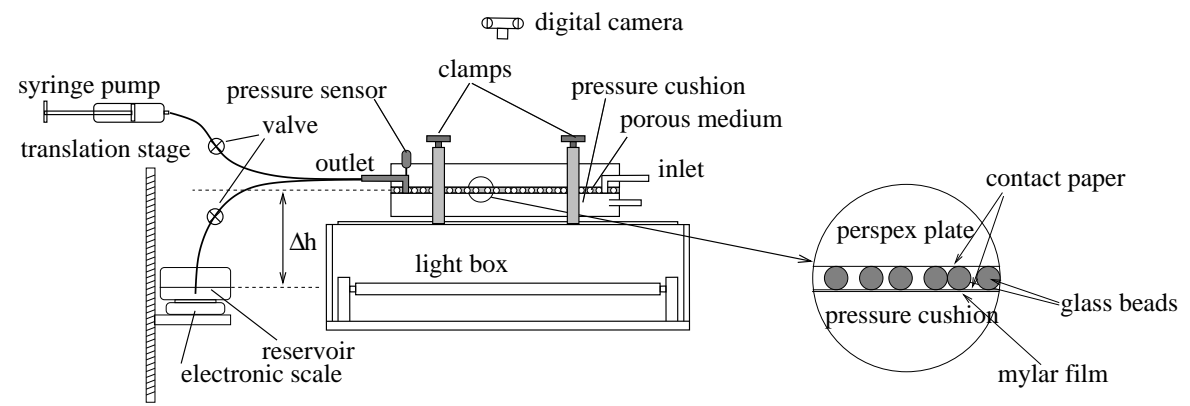

Figure 1. Sketch of the experimental rig used for the flow experiments. The two-dimensional porous model is clamped to a light-box and kept in place by a pressure cushion.

is initially completely filled with a wetting liquid, which is displaced by a non-wetting fluid (primary drainage).

The porous medium is made of a random mono layer of glass beads: Glass beads are poured on the sticky side of a contact paper (see Fig. 1). When the surface is completely filled with beads, excess beads are removed, leaving a random mono-layer of beads. The sticky paper is attached to a Plexiglas plate that has milled inlet and outlet channels $5 \mathrm{~mm}$ wide and $8 \mathrm{~mm}$ deep. The model is sealed off at the edges by silicon glue, thus forming a rectangular porous medium of width $W$ and length $L$.

When the model is sealed, another sticky paper is placed (glue down) on top of the glass bead layer. The resulting quasi two-dimensional porous medium can be filled with a liquid through the channels in the Plexiglas plate (see Fig. 1). The model is then placed (horizontally) with the beads down and clamped to a frame with an integrated pressure cushion. This pressure cushion ensures that the position of each bead is fixed and that the porous medium is always only one bead diameter thick (Fig. 1). The pressure cushion is filled with water and kept under hydrostatic pressure. The transparent model is placed and secured on top of a light-box. The porosity $\phi$ and intrinsic permeability $\kappa$ are measured for all the models. Average values are: $\phi=0.62 \pm 0.02$ and $\kappa=(0.017 \pm 0.002) \cdot 10^{-3} \mathrm{~cm}^{2}(\approx 1800 \pm 200$ Darcy $)$.

The wetting liquid is either a $80 \%-20 \%$ or a $90 \%-10 \%$ by weight glycerol-water solution, dyed with $0.1 \%$ Negrosine, and the invading fluid is air. At room temperature the wetting glycerol-water solution has a measured viscosity of $\mu_{\mathrm{w}} \approx 0.050 \mathrm{~Pa}$.s and $0.165 \mathrm{~Pa}$.s and a density of $\rho_{\mathrm{w}}=1209 \mathrm{~kg} \cdot \mathrm{m}^{-3}$ and $1235 \mathrm{~kg} . \mathrm{m}^{-3}$, respectively. The corresponding parameters for the non-wetting air are $\mu_{\mathrm{nw}}=1.9 \cdot 10^{-5}$ Pa.s and $\rho_{\mathrm{nw}}=1.20 \mathrm{~kg} \cdot \mathrm{m}^{-3}$. The viscous ratio is thus $M=\mu_{\mathrm{nw}} / \mu_{\mathrm{w}} \sim 10^{-4}$, 
which is low enough to render the pressure gradient negligible in the non-wetting liquid. The surface tension $(\gamma)$ between the two liquids is $\gamma=6.4 \cdot 10^{-2} \mathrm{~N} . \mathrm{m}^{-1}$ (Vedvik et al., 1998). Two different liquid mixtures are used in order to tune the viscosity contrast of the fluids while maintaining the wetting properties. The $80 \%-20 \%$ glycerol-water solution is used in the experiments presented in Sec. 3 while the 90\%$10 \%$ solution is used in the experiments presented in Sec. 4. In all experiments presented in Sec. 4 the temperature in the displaced wetting liquid is measured to enable control of the viscosity of the wetting liquid (and thus, of the capillary number).

The gathered data consists of pressure measurements, pictures of the displacement structure and the extracted liquid volume. The absolute pressure in the wetting liquid is measured at the outlet channel (Fig 1) using a Honeywell 26PCA or a Sensor Technics 26PC0100 Flow Through pressure sensor. Images are taken with a Kodak DCS 420 or a Nikon D200 digital camera. The extracted volume of wetting fluid is also recorded. All the data is collected and synchronized by a computer.

In this paper results from two model sizes are presented. All the experiments presented in Sec. 3 are performed on a model with $W=$ $200 \mathrm{~mm}$ and $L=350 \mathrm{~mm}$ (called the small model). In addition to this, in Sec. 4 we present results from a set of experiments for which $L=840 \mathrm{~mm}$ and $W=215 \mathrm{~mm}$ (called the large model).

Results from two fundamentally different types of experiments are presented: (i) quasi-static and (ii) constant flow rate experiments. In the first case, the flow is driven by slowly changing the pressure difference over the model. This is done by moving an open reservoir of the wetting liquid, which is connected to the models outlet channel, vertically with respect to the porous matrix. In the second case a preset constant flow rate is imposed by means of a syringe pump or a tailored gravity pump (Méheust et al., 2002; Løvoll et al., 2004) (see Fig. 1). Since the observed structure is highly dependent on the imposed flow rate, the latter method has the advantage that invasion happens at a constant well defined capillary number. This ensures that the length scales characteristic of the present flow regimes stay constant during the experiments (Løvoll et al., 2004; Toussaint et al., 2005).

\section{Saturation-Pressure relation: From capillary fingering to viscous fingering}

As mentioned in the previous section, two different types of primary drainage experiments were performed. In the first set of experiments, 

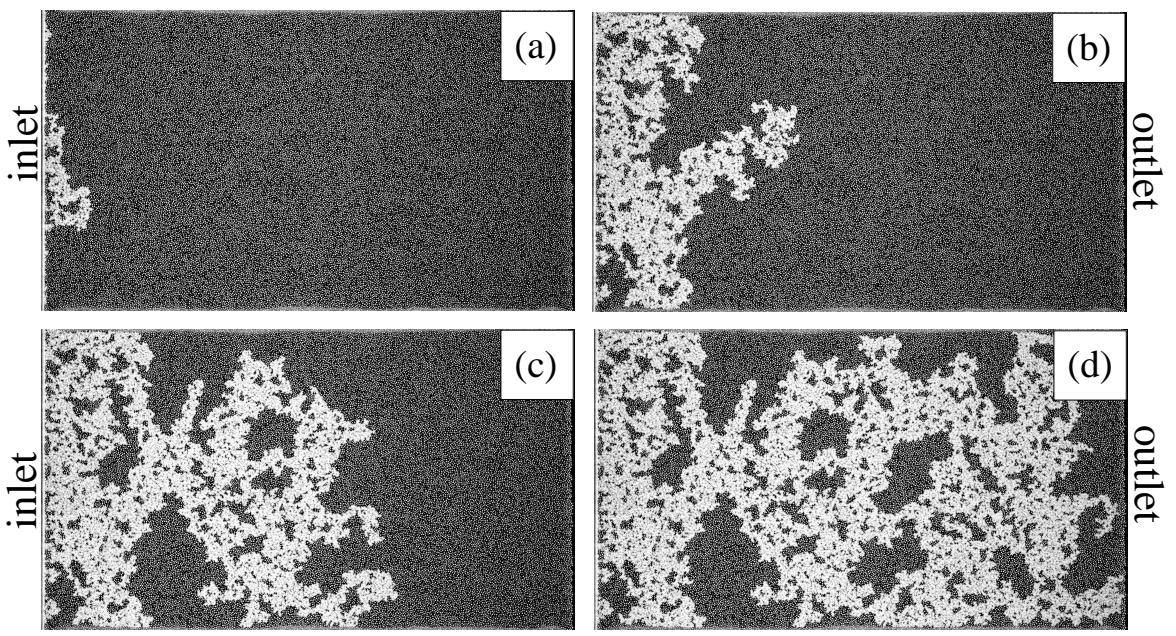

Figure 2. Snapshots showing the evolution of a quasi static drainage experiment. The wetting glycerol-water solution is dark and the air appear as white. The experiment is driven by slowly increasing the pressure difference over the system. The snapshots (a), (b), (c) and (d) are taken at $\sim 23.0,15.3,7.7$ and $0 \mathrm{~h}$ before breakthrough, respectively. The total time from start to breakthrough was $\sim 48 \mathrm{~h}$. The snapshots are indicated in the saturation-pressure curve on Fig. 3.

the pressure difference accross the model was controlled, and we performed slow (quasi-static) capillary fingering experiments.

In the second set of experiments, a faster constant flow rate was imposed to investigate the transition to viscous fingering. This was done on the same model as the quasistatic experiments, using a syringe pump. This allowed to perform constant flow rate $(\mathrm{Ca})$ experiments with the capillary number as tuning parameter.

These two sets of experiments were carried out in the same model (of dimensions $1 \mathrm{~mm} \times 200 \mathrm{~mm} \times 350 \mathrm{~mm}$ ), and will be presented in this section.

A third set of experiments, also at imposed capillary number, was performed, in which we analysed optically the invasion structure, simultaneously to the retention-saturation dependence. This was done in a larger model, of dimensions $1 \mathrm{~mm} \times 840 \mathrm{~mm} \times 215 \mathrm{~mm}$. The results of these experiments and their treatment to obtain a model of the velocity dependence of the curves will be presented in Sec. 4 .

\subsection{QuASI-STATIC EXPERIMENTS UNDER IMPOSED PRESSURE HEAD}

Fig. 2 shows a selected set of images from a single quasi-static drainage experiment. The sequence (a)-(d) shows the invader from the start of invasion up to just before "breakthrough", i.e. when the invader 
reaches the outlet channel. The invading cluster is typical of capillary fingering (Lenormand and Zarcone, 1985), it spans the whole system width and leaves behind trapped droplets of wetting fluid, of various sizes (only limited by the system size). The snapshots in Fig. 2 are also referred to in the corresponding saturation-pressure curve in Fig. 3. In this experiment the pressure drop over the system was changed slowly by keeping the atmospheric pressure at the inlet, while slowly lowering an open reservoir connected to the model outlet by tubes fully filled with the lowly compressible wetting fluid: this imposes a pressure drop corresponding to the hydrostatic pressure drop in the outlet tube (the vertically movable reservoir is indicated on Fig. 1). In order to minimize any additional pressure drop in the system due to fluid friction in the viscous fluid (dynamic effects), the reservoir is displaced vertically by small increments, and kept at rest between two successive increments until the fluids are not flowing anymore. At this point, the pressure at the setup outlet is constant and related to the atmospheric pressure $P_{\mathrm{a}}$ and to the altitude of the reservoir (referenced with respect to the outlet's altitude) through the law of hydrostatic pressure. As the setup inlet is open to the air, the pressure is $P_{\text {a }}$ there as well, so the deviation of the pressure at the outlet from the atmostpheric pressure also happens to be the pressure head between the setup inlet and outlet. Since the viscosity of the air is negligible with respect to that of the liquid, that pressure head is also the sum of the capillary pressure and of the pressure head through the viscous liquid. This way of driving the system is what we call "quasi-static". As described in the previous section, we measure the amount of liquid extracted from the model and the pressure at the setup outlet (gauge pressure sensor). From these mass measurements the saturation of the non-wetting liquid $\left(S_{\text {nw }}\right)$ is calculated as a function of time. By combining the calculated saturation with pressure measurements, a standard saturation-pressure curve is obtained. Pressure-saturation data from the experiment shown in Fig. 2 are plotted in Fig. 3.

As can be seen in Fig. 3, the pressure measurements exhibit notable fluctuations during the drainage process.

The sudden (random) jumps seen around the plateau corresponding to the hydrostatic pressures imposed by the reservoir happen during the dynamic stages, that is just after each displacement increment of the reservoir and before the flow has ceased, as the fluid interface (invasion front) penetrates new pores. During these dynamic stages, the measured pressure is different from that imposed at the setup outlet by the reservoir under static conditions. In order to invade a pore, the capillary pressure has to exceed the capillary threshold pressure for that pore throat. As this happens the capillary pressure decreases 


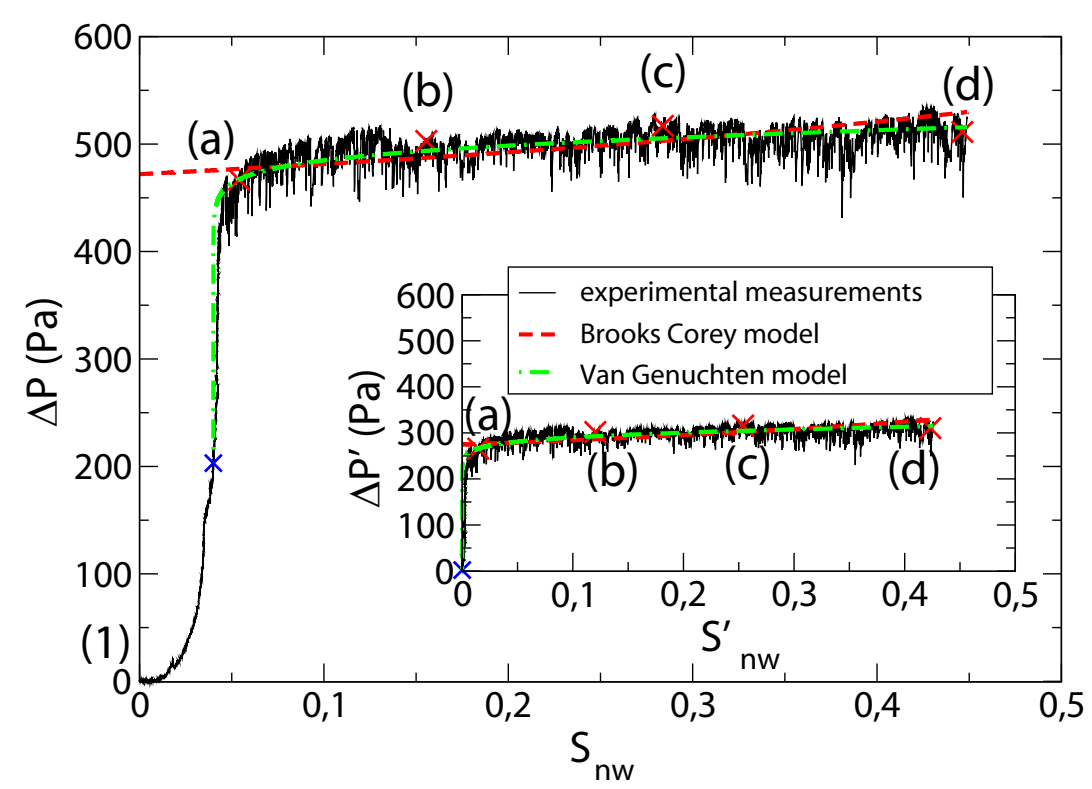

Figure 3. Pressure-saturation (retention) curve from a quasi-static primary drainage experiment.The plotted pressure is the pressure difference over the model (from invasion front to outlet channel). The pressure was measured with a single gauge sensor in the outlet channel where the pressure recorded before the invasion process starts is used as reference. The plotted data is taken from the experiment shown in Fig. 2, the corresponding images are indicated by red crosses on the graph (a)-(d) from left to right. The subplot indicates the modified pressure and saturation, with a reference taken at the moment indicated by the blue cross, after the invader has filled a grain free buffer region in the Hele-Shaw cell, when the interface between the two fluids reaches the boundary of the granular medium. The two curves plotted are Brooks-Corey (dashed) and Van Genuchten (dot-dashed) models fitted on the data in the subplot - and reported on the main figure. This saturation-pressure curve seems consistent with a behavior typical of soil mechanics.

rapidly, the pore is filled with the invading fluid and the fluid interface in the surrounding pores retracts in order to adjust to the new capillary pressure. The size of these pressure jumps is random since the capillary thresholds are randomly distributed (Måløy et al., 1992; Furuberg et al., 1996). All pore necks in the system have an associated capillary threshold pressure. These threshold pressures are determined by the local pore geometry, the surface tension between the fluids, and the local wettability. So for a given porous medium and fluid pair the thresholds will have a given distribution. During drainage a subset of this distribution is probed before the invader percolates the sys- 
tem (Auradou et al., 1999; Schmittbuhl et al., 2000; Auradou et al., 2003). If the experiment is driven by slowly increasing the pressure difference between the air and the liquid phase over the model, the invasion process starts once the pressure has reached the lowest pressure in the capillary threshold pressure distribution. For the data plotted in Fig. 3 this corresponds to the steep increase in pressure as air start invading the system. As the pressure is slowly increased further a larger portion of the threshold distribution becomes available for invasion, and while the pressure slowly changes, the non-wetting fluid invades the system and the saturation of non-wetting fluid increases, until the air percolates the system at breakthrough. Therefore, for "quasi-static" experiments, relatively large changes in saturation occur for small pressure increases once the pressure is established at values well inside the capillary threshold distribution.

One can directly compare the pressure saturation curve of these quasistatic tests in grain filled Hele Shaw cells to standard models from the hydrology literature: denoting the effective water saturation as

$$
S_{e}=\frac{S_{w}-S_{w r}}{1-S_{w r}}=\frac{1-S_{n w}-S_{w r}}{1-S_{w r}}
$$

where $S_{w r}$ is the residual water saturation, the Brooks-Corey model (Brooks and Corey, 1964) is normally written as

$$
p_{c}=p_{d} S_{e}^{1 / \lambda}
$$

where $p_{d}$ is the air entry pressure. A least-square fit of the reduced data to this model is shown in Fig. 3, and provided the following fit parameters: $p_{d}=275 \mathrm{~Pa}, \lambda=3.1$, and $S_{w r}=0$. Typically, for soils, $\lambda$ stands between 0.2 and 3 , the highest values corresponding to highly non-uniform grain-size distribution. The fact that we obtain a value close to the higher naturally occurring values, is consistent with the fact that the nearest neighbor distance, in such layers of beads put at random on a sticky plate, is certainly highly non-uniform compared to a three-dimensional packing of grains stacked under gravity. Given the value of the interfacial tension $\gamma$, the entrance pressure corresponds to a minimal curvature in the largest pore necks of $r_{\|}=\gamma / p_{d}=0.25 \mathrm{~mm}$, which is of the order of a quarter of bead size: this is consistent with the expected order of magnitude for such a packing. The fact that $S_{w r}$ is obtained at its smallest possible value, 0 , can be explained by the fact that apart from the large fluctuations, no trend of any upward curvature of the pressure-saturation curve can be seen in Fig. 3. 
Another common model for such curves in the Van Genuchten retention function (Van Genuchten, 1980),

$$
p_{c}=\frac{1}{\alpha_{v g}}\left(S_{e}^{\frac{n}{1-n}}-1\right)^{\frac{1}{n}}
$$

A fit to this model is shown as the dash-dotted curve in Fig. 3, and leads to Van Genuchten parameters $\alpha_{v g}=0.00029 \mathrm{~Pa}^{-1}, S_{w r}=1$, and $n=9.9$. The value of $\alpha_{v g}$ obtained is consistent with that of a soil, the value of $\mathrm{n}$ is a few times larger than the one usually obtained for three-dimensional natural soils. This can be presumably attributed to differences in the packing, and notably the fact that the transition from the steeply growing part of the curve, up to point (a), into a plateau with fluctuations, is quicker than for many natural three-dimensional soils presumably due to the fact that only one diameter of beads is represented in the packing.

From a general point of view, it is interesting to note that this two dimensional medium presents a quasi-static pressure-saturation curve (and model parameters) somewhat similar to what could be expected for its three dimensional counterpart, and presumably represents well the processes at play in tests on opaque three dimensional media.

\subsection{EXPERIMENTS UNDER FINITE IMPOSED FLOWRATE}

As mentioned, we have also driven the system at constant capillary number by means of a syringe pump. In this case a constant flow rate is imposed, and the pressure over the model will at any time be the pressure needed to maintain the flow rate. Fig. 4 shows the saturationpressure data curve for syringe pump driven experiments at different imposed flow rates. The black curve in Fig. 4 shows a slowly driven experiment, at $C a=0.01$. It shows the same initial pressure buildup in the beginning of the experiment as for the "quasi-static" experiment (Fig. 3). When the average capillary threshold pressure is reached the pressure just fluctuates more strongly around this constant value during the whole drainage process. The two other curves shown in Fig. 4 present similar results for faster syringe pump driven experiments. These curves still show the random pressure fluctuations caused by the variation of capillary pressure thresholds. Additionally, compared to quasistatic drainage curves, the slope of the drainage curve is "reversed" by the boundary conditions of imposed flux: after the initial pressure buildup, the pressure is steadily decreasing while the air satu-

ration increases. This feature was also observed with different boundary conditions in some numerical studies of dynamic fingering (Dahle et al., 2005). As already known and shown in other studies (Hassanizadeh 


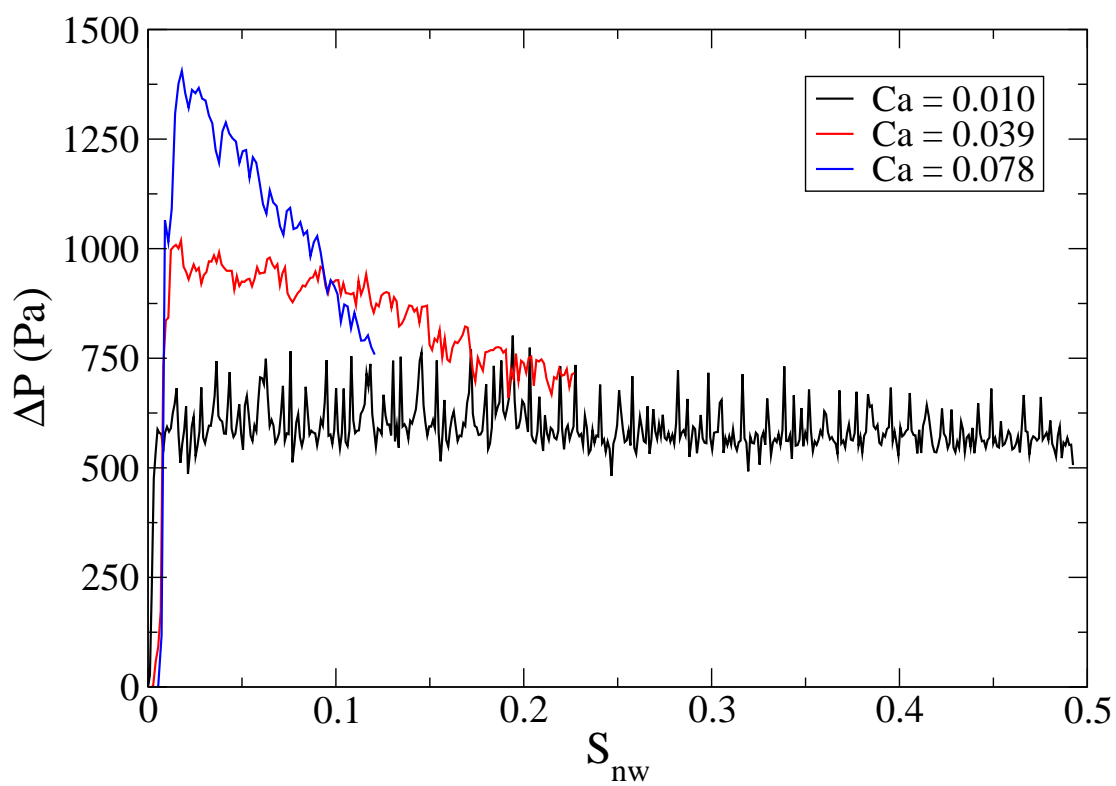

Figure 4. Saturation-pressure curves from three primary drainage experiments on the small model, driven at constant flow rate (constant $\mathrm{Ca}$ ) by a syringe pump. $S_{n w}$ is the saturation of the system in the non-wetting fluid (air), related to the saturation in wetting fluid (water-glycerol) $S_{w}$ by $S_{n w}=1-S_{w}$. The total duration of the drainage experiments are $\sim 2.4 \mathrm{~h}, \sim 10 \mathrm{~min}$. and $\sim 3 \mathrm{~min}$ for $\mathrm{Ca}=0.010$, 0.039 and 0.078 , respectively.

et al., 2002; Dahle et al., 2005), we observe here that for a given porous medium there is no one-to-one relation between the saturation and the pressure over the system. Boundary conditions and dynamic effects have a significant impact on the pressure vs. saturation relation. In the next section we shall look at dynamic effects more closely.

\section{Relating capillary number, saturation, pressure and system size}

We now turn to the results from experiments carried at different imposed flow rate on on the large model $(1 \mathrm{~mm} \times 215 \mathrm{~mm} \times 840 \mathrm{~mm})$. Dynamic capillary pressure as measured in retention experiments (Hassanizadeh et al., 2002) is often defined as the total pressure difference over the porous medium $(\Delta P)$, or some spatial average of the pressure over one of the present phases (Dahle et al., 2005) (see discussion in Sec. 5). The total pressure difference is a combination of capillary pressure along the invasion front $\left(P_{\mathrm{c}}\right)$ and the pressure head caused by 

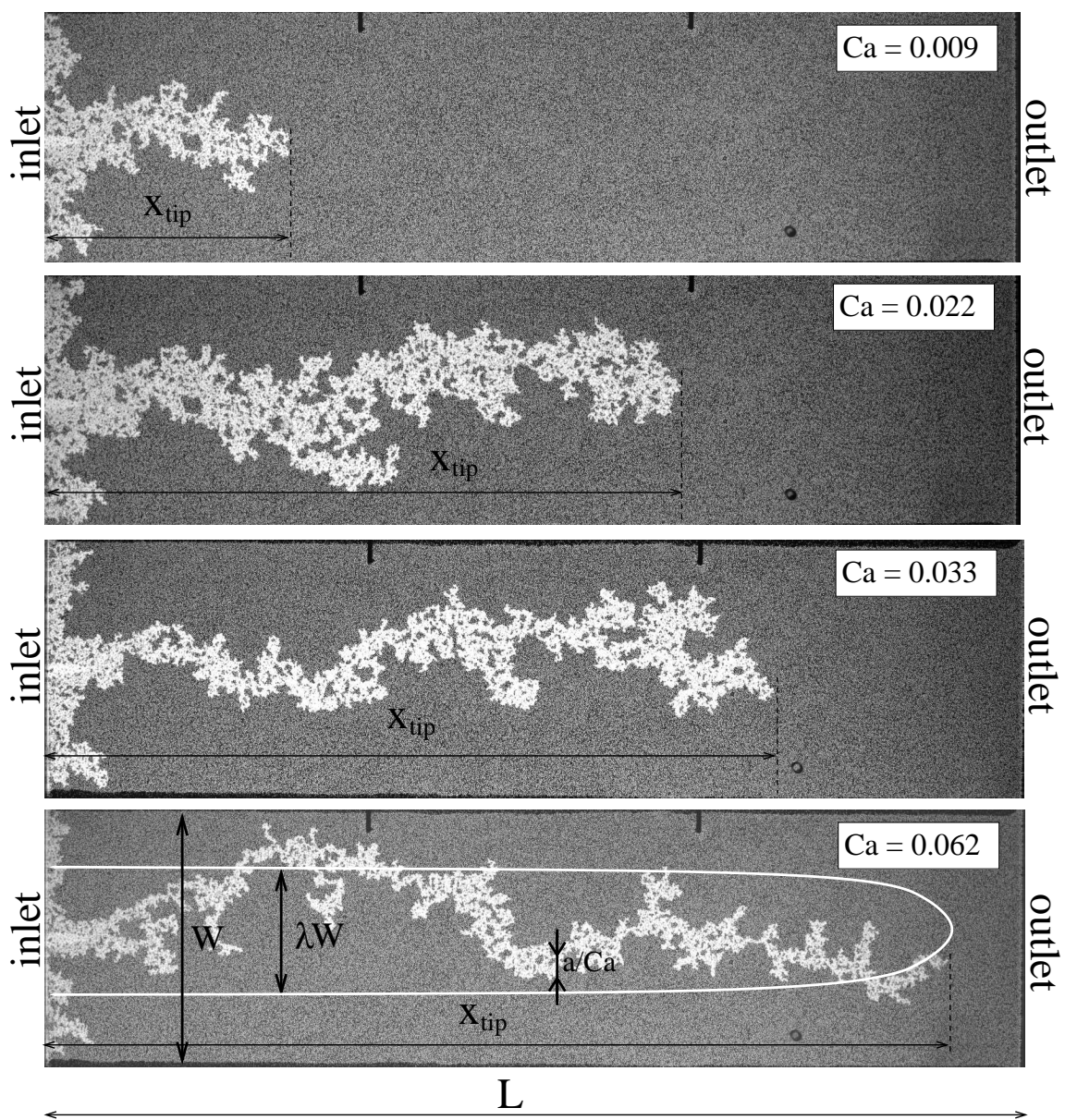

Figure 5. Images of experiments taken at different withdrawal rates (expressed by the capillary numbers). The viscous pressure difference over the model is $P \sim 490 \mathrm{~Pa}$ in all the pictures. The invasion structure is initiated in the center of the flow cell by creating a small "notch" in the porous medium at the center of the inlet. On the lower image relevant length scales are indicated.

viscous effects $\left(\Delta P_{\mathrm{v}}\right)$ (Washburn, 1921)

$$
\Delta P=P_{\mathrm{c}}+\Delta P_{\mathrm{v}}
$$

In drainage the capillary pressure is essentially unaffected by the speed of the flow and system size (Méheust et al., 2002), except in details, since the pores invaded are not exactly the same. (This is however not the case in fast imbibition, see (Weitz et al., 1987)). The order of magnitude of this capillary pressure does not change in time and as demonstrated in Sec. 3 it can be treated as randomly fluctuating 


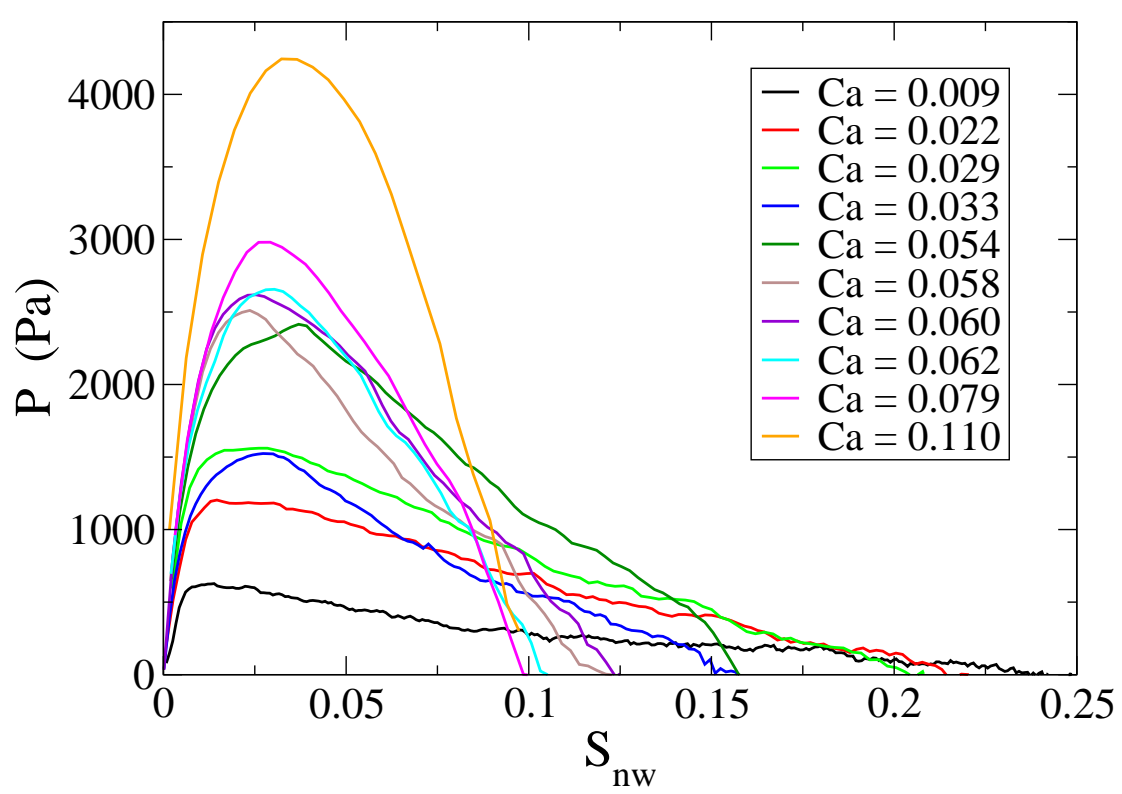

Figure 6. Pressure (see Eq. 4) as a function of the non-wetting fluid saturation $\left(S_{\mathrm{nw}}\right)$ for some experiments on the large model. The initial pressure buildup is due to inertia in the liquid. The effect is explained in Appendix A.

around a well defined average value. However, the viscous part $\left(\Delta P_{\mathrm{v}}\right)$ is dependent on finite size effects and invasion speed. In the following, the reported measured pressure $(P)$ is therefore equal to the viscous pressure drop:

$$
P=\Delta P_{\mathrm{v}}
$$

It is obtained from our measurements by using the pressure just before break through $\left(t_{\mathrm{bt}}\right)$ as reference null pressure $P\left(t_{\mathrm{bt}}\right) \equiv 0$.

In Fig. 5, snapshots of the invasion structure are shown for four different drainage experiments. The experiments are all performed at constant flow rate and the snapshots are such that the viscous pressure drop across the cell is the same in all snapshots. These pictures do also illustrate how the saturation of the non-wetting liquid changes with capillary number. As the invasion rate increases, the invasion structure gets thinner, which again leads to lower saturation of the invading nonwetting liquid. The pressure vs. air-saturation $\left(S_{\mathrm{nw}}\right)$ plots for these drainage experiments are shown in Fig. 6. After the initial pressure buildup, these experiments show the same behavior as the two "fast" syringe driven experiments in the previous section (Fig. 4).

If the system is big enough, viscous effects will always dominate the problem at larger length scales. The crossover length scale such 
that the viscous pressure drops between two points separated by a distance larger than this scale dominate over capillary threshold fluctuations, is given by Eq. (2), $l_{\mathrm{c}}=a /$ Ca. Above this length scale, the displacement dynamics is in the viscous fingering regime and it will take place in narrow channels and branched loopless structures, as shown in Fig. 5 (Løvoll et al., 2004; Toussaint et al., 2005). At scales below this, capillary fluctuations dominate the dynamics, and the resulting structure is characteristic of the capillary fingering regime, with droplets of wetting fluid remaining trapped in loops of the invading nonwetting fluid. Looking at Fig. 5, four experiments at different speeds and at the same $P$, it is obvious that the viscous pressure drop is related to the position of the most advanced finger and the capillary number. Earlier studies have also shown that the invasion structure is screened by the advancing invasion front and that the propagation speed of the invasion front is fairly constant (Løvoll et al., 2004; Toussaint et al., 2005). The process is thus stationary in a referential attached to the advancing finger tip. All the invasion happens in a zone close to this tip. As was obtained in a detailed analysis of the pressure field, measuring at several points around the invader in each experiment (Løvoll et al., 2004), the pressure is roughly constant behind the finger tip, and the viscous pressure gradient in front of the invasion structure is essentially constant. The viscous pressure is thus (to the first order) a linear function of the distance from the outlet to the most advanced finger:

$$
P=\overline{\nabla P}\left(L-x_{\text {tip }}\right)
$$

where $\overline{\nabla P}$ is the average viscous pressure gradient and $x_{\text {tip }}$ is the tip position of the most advanced fingertip (indicated on Fig. 5). $\overline{\nabla P}$ can be approximated by Darcy's law:

$$
\overline{\nabla P}=\frac{\mu_{\mathrm{w}} v_{\mathrm{f}}}{\kappa}
$$

Consequently, we can write the pressure change caused by viscosity effects as:

$$
P=\frac{\mu v_{\mathrm{f}}}{\kappa}\left(L-x_{\mathrm{tip}}\right)=\frac{\gamma \mathrm{Ca}}{a^{2}}\left(L-x_{\mathrm{tip}}\right),
$$

where we have used the expression of the capillary number, Eq. (1). This allows to express the ratio of $x_{\text {tip }}$ to the system length $L$ as a function of pressure and capillary number:

$$
1-\frac{x_{\mathrm{tip}}}{L}=\frac{a^{2}}{\gamma L \mathrm{Ca}} P
$$

where we recall that $P$ is the measured viscous pressure drop over the system (see Eq. (4)). 


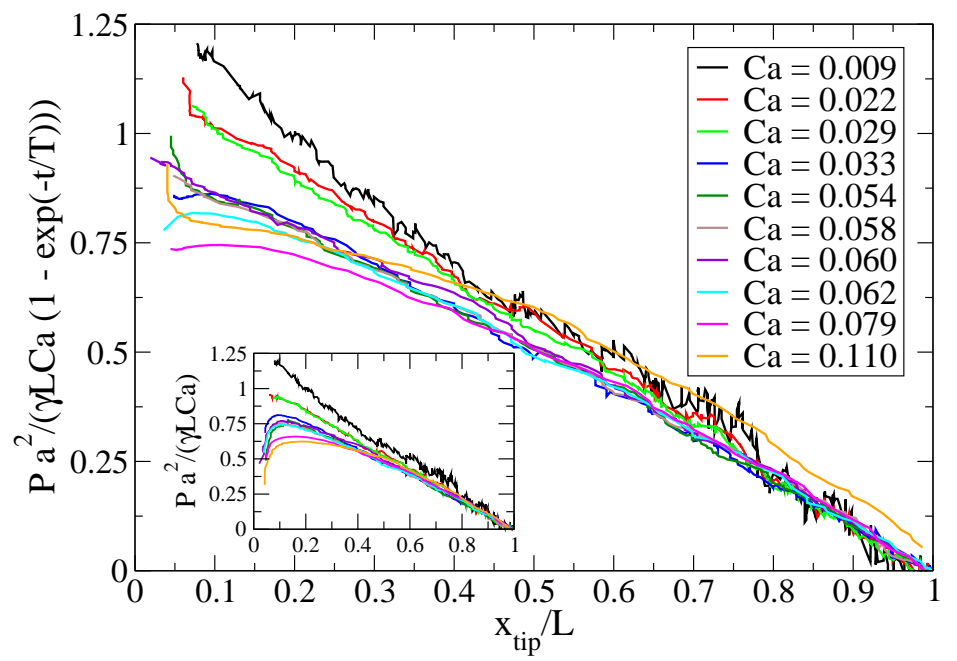

Figure 7. Data collapse of pressure data as function of $x_{\text {tip }} / L$, where the initial pressure buildup is corrected with the method outlined in Appendix A using T=114 s. The inset show scaled pressure data without initial pressure correction.

In the inset of Fig. 7, where the rescaled pressure $a^{2} P /(\gamma L C a)$ is plotted as a function of the reduced finger tip position $x_{\text {tip }} / L$ for a range of capillary numbers. The pressure drop across the model can also be corrected for the inertia of the fluid during the initial acceleration. In Appendix A we show how these initial inertial effects lead to a multiplicative factor $\left(1-e^{-t / T}\right)$ for the time dependant pressure, where $T$ is the time characteristic of the initial pressure buildup. A way to correct for these inertial effects is then, after the determination of $T$ by a least square fit, to represent the corrected pressure $P /\left(1-e^{-t / T}\right)$ rather than the measured pressure. This is shown in Fig. 7. Based on the argument above, we introduce the dimensionless reduced pressure:

$$
P^{*} \equiv P \frac{a^{2}}{\gamma L \mathrm{Ca}}\left(1-e^{-t / T}\right)^{-1}=1-\frac{x_{\text {tip }}}{L}
$$

Using our knowledge of the morphology of the invading cluster in the transition from viscous- to capillary-fingering (Løvoll et al., 2004; Toussaint et al., 2005), a relation for the non-wetting liquid saturation $\left(S_{\text {nw }}\right)$ as a function of finger tip position $x_{\text {tip }}$, capillary number $(\mathrm{Ca})$ and the width of the system $W$ can also be derived. This is done by counting the number of invaded pores $N_{\text {inv }}$ as a function of $x_{\text {tip }} / L$. Earlier studies (Løvoll et al., 2004; Toussaint et al., 2005) have shown that the invasion typically takes place in a limited region in the center of the channel. The width of this region is $\lambda W$, where $W$ is the width of the 
system and $\lambda \simeq 0.4$. Above this scale, we have a linear channel where the displacement structure could be considered homogeneous. Inside this channel (of width $\lambda W$ ), two flow regimes are present depending on the length scale considered. At large scales (between $\lambda W$ and $l_{\mathrm{c}}$ ) the flow structure is that of viscous fingering, which is a treelike structure with branches and no loops. The viscous fingering structure is a fractal with a fractal dimension $D_{\mathrm{v}} \simeq 1.53$ (Toussaint et al., 2005). At smaller scales capillary forces dominates the flow process and the displacement structure is that of capillary fingering. Capillary fingering is also fractal, with fractal dimension $D_{\mathrm{c}} \simeq 1.83$ (Wilkinson and Willemsen, 1983; Lenormand and Zarcone, 1985). The relevant length scales in the problem are sketched in Fig. 5. The following argument is thus valid when:

$$
a \ll \frac{a}{\mathrm{Ca}}=l_{\mathrm{c}} \ll \lambda W
$$

When the most advanced finger tip is at $x_{\text {tip }}$, the invasion has

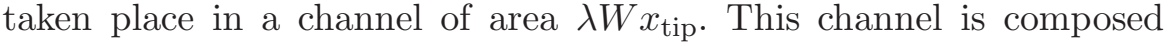
of $x_{\text {tip }} /(\lambda W)$ square zones of linear size $\lambda W$. In each of these zones the invading viscous fingering structure goes through $(\lambda W \mathrm{Ca} / a)^{D_{v}}$ square boxes of linear size $a / C a$. And in each such box, the invader occupies on average $[(a / \mathrm{Ca}) /(\alpha a)]^{D_{c}}=(\alpha \mathrm{Ca})^{-D_{c}}$ pores of linear size $\alpha a$, with $\alpha$ a geometrical pre factor, of order of magnitude 1 (indicating that the average pore volume is not exactly $a^{3}$ but rather $\left.(\alpha a)^{3}\right)$. The total number of invaded pores is thus:

$$
\begin{aligned}
N_{\text {inv }}\left(x_{\text {tip }}\right) & =\frac{x_{\text {tip }}}{\lambda W}\left(\frac{\lambda W \mathrm{Ca}}{a}\right)^{D_{\mathrm{v}}}(\alpha \mathrm{Ca})^{-D_{\mathrm{c}}} \\
& =\frac{x_{\text {tip }}(\lambda W)^{D_{\mathrm{v}}-1}}{a^{D_{\mathrm{v}}}} \alpha^{-D_{\mathrm{c}}} \mathrm{Ca}^{D_{\mathrm{v}}-D_{\mathrm{c}}}
\end{aligned}
$$

The total volume accessible to the fluid is $W L a \phi$ and the average pore volume is $(\alpha a)^{3}$. The saturation of the non-wetting fluid, $S_{\mathrm{nw}}$, can thus be written as:

$$
\begin{aligned}
S_{\mathrm{nw}} & =\frac{N_{\text {inv }}(\alpha a)^{3}}{W L a \phi} \\
& =\frac{x_{\text {tip }}}{L} \frac{a^{2-D_{\mathrm{v}}}}{\phi} \frac{(\lambda W)^{D_{\mathrm{v}}-1}}{W} \alpha^{\left(3-D_{c}\right)} \mathrm{Ca}^{D_{\mathrm{v}}-D_{\mathrm{c}}}
\end{aligned}
$$

This relation can be inverted to obtain a dimensionless reduced saturation $S^{*}$ which is a reduced variable of the tip position, $x_{t i p} / L$. It relates to the dimensional saturation of the wetting fluid, $S_{\mathrm{nw}}$, according to:

$$
S^{*} \equiv \frac{x_{\mathrm{tip}}}{L}=S_{\mathrm{nw}} \alpha^{\left(D_{c}-3\right)} \frac{\phi}{\lambda^{D_{\mathrm{v}}-1}}\left(\frac{W}{a}\right)^{2-D_{\mathrm{v}}} \mathrm{Ca}^{D_{\mathrm{c}}-D_{\mathrm{v}}}
$$


Based on Eq. (9) and (13) we predict that $P^{*}$ and $S^{*}$ are related through the relation:

$$
P^{*}=1-S^{*}
$$

This is indeed the case to a large extent, as shown in Fig. 8. The satisfactory character of this data collapse for the reduced pressure drop versus reduced saturation at various speeds can be seen when comparing Fig. 8 to the raw data presented in Fig. 6 .

In addition, the above arguments can be applied to the particular state $P^{*}=0$, i.e. at breakthrough, when the invading nonwetting fluid reaches the outlet, at $x_{t i p}=L$. The saturation reached at this time gives the final saturation which can be retrieved before the invader reaches the outlet, and thus gives an estimate of the efficiency of the invasion process before breakthrough. This point corresponds to the prediction $S^{*}=1$, i.e. according to Eq. (13), to a final non wetting saturation $S_{\text {nw }}$ at breakthrough

$$
S_{\mathrm{nw}}\left(t_{b t}\right)=\alpha^{\left(3-D_{c}\right)} \frac{\lambda^{D_{\mathrm{v}}-1}}{\phi}\left(\frac{a}{W}\right)^{2-D_{\mathrm{v}}} \mathrm{Ca}^{D_{\mathrm{v}}-D_{\mathrm{c}}} .
$$

and a residual saturation $1-S_{\mathrm{nw}}$ that reads accordingly. This prediction for the final saturation as a function of the speed of the invasion process $\mathrm{Ca}$ - is shown in the inset of Fig. 8 as a dashed curve. The measurements of the final invader saturation at breakthrough for experiments carried out at various capillary numbers, represented as the crosses, match well with this prediction.

\section{Discussion}

The theory leading to the scaling relation between pressure and saturation in Eq. (14) is valid for a limited range of capillary numbers, for high viscous contrast and for systems without gravity. The limits in capillary numbers are given in Eq. (10). We believe that the upper limit in capillary number is $\mathrm{Ca} \approx 1 / 10$ which implies that our largest reported capillary number $\mathrm{Ca}=0.11$ is close to this limit. For higher capillary numbers, the width of the viscous fingers is close to the pore scale and there is no capillary fingering structure at smaller scales. This would imply that the saturation is independent of the capillary number at higher flow rates and is constant for all $\mathrm{Ca}$ higher than the ones satisfying Eq. (10). This observation is consistent with the measured break through saturation shown in the inset of Fig. 8 where the saturation level seems to be fairly constant for capillary numbers above $\sim 0.1$. At the other end of the scale, it is the size of the system that sets the limit. 


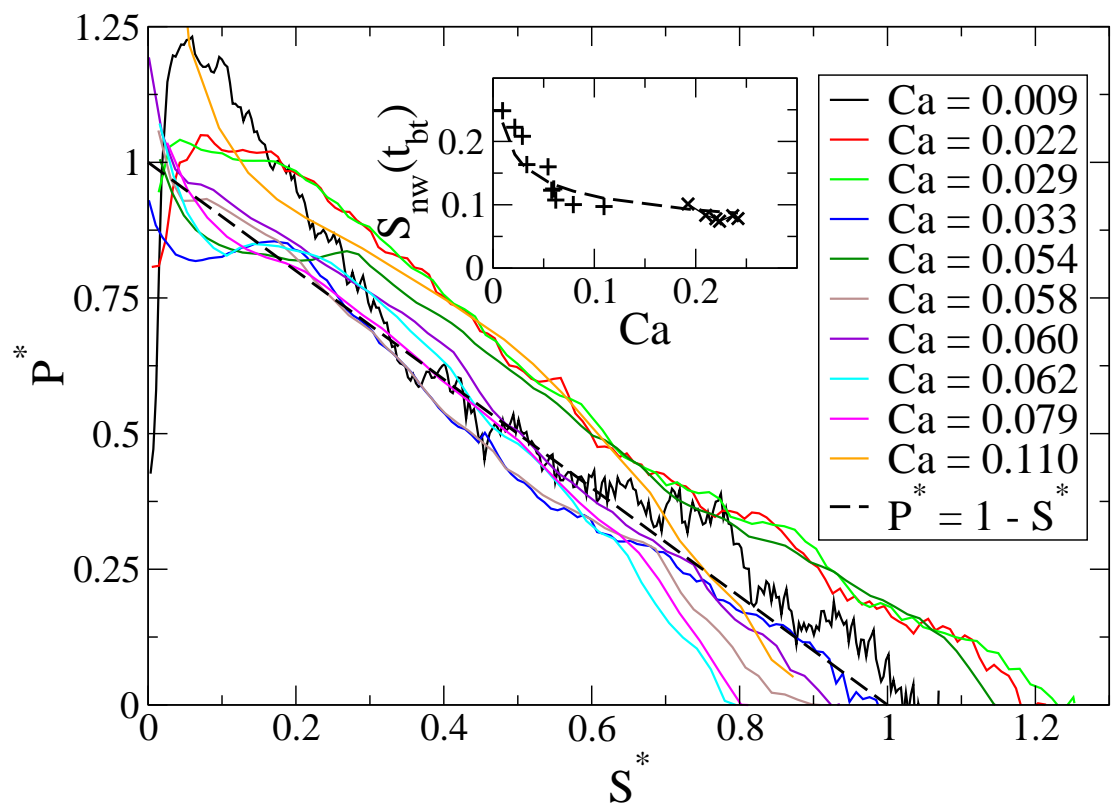

Figure 8. Data collapse of saturation versus pressure curves from experiments performed at capillary numbers between $9 \cdot 10^{-3}$ and $1.1 \cdot 10^{-1}$. The collapse is obtained by plotting $P^{*}$ as a function of $S^{*}$. The collapse is consistent with the prediction in Eq. (14); it is indicated by the dashed line. Compare this to the original data shown in Fig. 6. The only "free parameter" in the data collapse is the geometrical pre-factor $\alpha, \alpha=0.75$ is used in the collapse. The inset shows the saturation of the non-wetting fluid $\left(S_{\mathrm{nw}}\right)$ at breakthrough time $\left(t_{\mathrm{bt}}\right)$ as a function of the capillary number. The dashed line is the breakthrough saturation predicted by Eq. (15).

When the capillary number is low enough for the crossover length scale to reach the system width $\left(l_{\mathrm{c}} \approx \lambda W\right)$ (see Fig. 2), there is no crossover from capillary to viscous fingering. Therefor a constant breakthrough saturation will be obtained. In our case this corresponds to $\mathrm{Ca} \approx 1 / 100$ which is of the order of the smallest presented capillary number.

It should also be noted that the invading cluster is fractal for all capillary numbers and the saturation will depend on the width $W$. At finite flow rates the system size determines the importance of viscous effects (dynamic effects). A given capillary number Ca will result in a crossover length scale $l_{\mathrm{c}}=a / \mathrm{Ca}$. Above this length scale viscous effects will dominate the problem. This observation raises a question on the general validity of the representative elementary volume (REV) assumption commonly used in Darcy scale modeling.

The data collapse of experimental measurements presented in Fig. 8 displays a certain dispersion. The quality of this collapse becomes evident when it is compared to the raw saturation-pressure data shown 
in Fig. 6. Fig. 7 shows that the collapse of the pressure data is good, so the main scatter in the collapse of Fig. 8 results from the saturation data. Even if there is scatter in the saturation data, we will claim that no systematic trend is visible. The experiments are carried out on disordered media, and the morphology of the invasion structures are statistically fractals. On such a small systems, noise is expected. For these model systems there are also boundary effects at the inlet and outlet. The arguments leading to Eq. (12) assume that the process is stationary, and they do not take boundary effects into account. If one looks at Fig. 5 it is evident that there are boundary effects near the inlet channel and that the saturation there is different from the saturation further into the model. In addition to this, the saturation in the frontal region is different from the stationary part left behind the advancing front, so when the invasion front reaches the outlet channel, the saturation will be lower in the vicinity of that channel than the saturation away from it. Earlier studies (Løvoll et al., 2004; Toussaint et al., 2005) have shown that the size of the active frontal region is of the order of the system width. At break through there is therefore a region at a distance $\propto W$ where the saturation is lower than in the "completely invaded" parts of the system. It is therefore crucial that the system is large enough to average out these fluctuations and to minimize end effect. So, when designing such an experiment, we would suggest that one ensures that $L \geq 4 W$ and in addition the system has to be wide enough so that Eq. (10) allow for a sufficiently large range of available capillary numbers.

As said earlier, the pressure (or dynamic capillary pressure) is often measured between two points, one in one phase, the other in the other one. Commonly, this is done practically at the inlet and outlet. However, some authors (Hassanizadeh et al., 2002; Dahle et al., 2005) define the dynamic capillary pressure as being the difference between the (spatial) average pressure in one phase, and the average pressure in the other one. Although this definition is easy to use in a numerical system, it is difficult to use in practice in an experimental system, where the pressure in the two phases is normally known only at a limited set of measurement points. We have measured (Løvoll et al., 2004; Toussaint et al., 2005) that the pressure in the wetting liquid is roughly spatially linearly decreasing in front of the invading tip, and roughly constant (equal to $P_{c}$ ) on the sides behind the tip - this is an approximation, see (Løvoll et al., 2004) for details of the pressure modeled and measured around the air fingers. The pressure in the continuous air phase is roughly constant. Consequently, the spatial average of the air pressure is $P_{a t m}$, and the average pressure in the wetting phase is dominated by 
the pressure ahead of the tip, i.e. the term

$$
\int_{x_{t i p}}^{L} d x\left(P_{a t m}+P_{c}+P \cdot \frac{x-x_{t i p}}{L-x_{t i p}}\right) /\left(\int_{x_{t i p}}^{L} d x\right)=P_{a t m}+P_{c}+P / 2 .
$$

Here, this difference between the average pressures in both phases average should be around

$$
P / 2+P_{\mathrm{c}},
$$

when the measured total variation across the cell is

$$
\Delta P=P+P_{\mathrm{c}}:
$$

Both should be qualitatively the same in terms of dependence on the flow speed, system size, etc. As with the measurements presented here, the "dynamic capillary pressure" is expected to be system size dependent. Whenever there is flow, there are dynamic effects present, and the pressure gradient is a priori known to be non zero $(\mathrm{Ca} \neq 0 \Leftrightarrow \nabla P \neq 0)$. In our case, the invading structure is screened from the viscous pressure field by the active zone near the most advanced finger (Løvoll et al., 2004). This implies that in the frontal region there is a spatial trend in the capillary pressure along the fingers: the capillary pressure in the fingertips (where invasion is happening) is larger than further back. This has been reported in earlier studies (Løvoll et al., 2004), and the effect is also present in the classic Saffman-Taylor solution (Saffman and Taylor, 1958).

It is also interesting to note that the models proposed by the authors of Refs. (Hassanizadeh et al., 2002; Dahle et al., 2005) include a linear dependency of the time derivative of the saturation $(\partial S / \partial t)$ in the "dynamic capillary pressure" function. It is important to mention that for a system of volume $V$ and porosity $\phi, \partial S / \partial t=Q \phi / V$, so that $\partial S / \partial t \propto$ Ca. This effect, reported by (Hassanizadeh et al., 2002; Dahle et al., 2005), is indeed a viscous effect (Niessner and Hassanizadeh, 2008), and for high viscous contrasts the proportionality factor has been derived in Sec. 4.

In the scaling theory developed in Sec. 4, the invader morphology at small scales is used to predict "Darcy scale" behaviour. We therefore believe that this type of argument could be very useful for Darcy scale modeling. Our results also demonstrate that for flow conditions where the morphology of the invader is fractal, the saturation-pressure relation is system size dependent. So in a sense there is no such thing as "continuum scale" in fractal systems. Our approach is thus rather a way to understand the problem at the relevant scales than a model for large scale continuum modeling. In real reservoir modeling one also has to consider effects and problems not included in our theory. At larger 
length scales, inhomogeneities in the porous media are expected and will be important. This implies that the relevant length scales will be different in the different parts of the system. The invasion speed and thus the capillary number can vary in space and time. In addition, in three dimensional systems, gravity and fluid density differences can be very important. When dealing with systems where gravity is important, viscous effects will still be important (Hill, 1952; Saffman and Taylor, 1958; Méheust et al., 2002) and in this class of problems also the ratio of the relevant forces can be used to predict the relevant length scales of the problem (Wilkinson, 1984; Birovljev et al., 1991; Méheust et al., 2002). Another limitation in our study is the high viscous contrast between the phases. In this respect we would like to point out that

there will generally be a high viscous contrast in systems where one of the phases is a gas, and that similar arguments have been successfully applied to systems where the viscous contrast were smaller (Frette et al., 1997; Aker et al., 2000).

In contrast to models used to describe standard water retention tests in soil samples, there are no semipermeable membranes at the inlet- and outlet-channel in the models used in this study. This makes it impossible to statically increase the pressure over the sample in order to reach the low water saturations commonly seen in published retention curves (Leverett, 1941; Brooks and Corey, 1964; Van Genuchten, 1980; Lenhard et al., 1993; Hassanizadeh et al., 2002). This choice for our models was motivated by the idea that the lack of semi permeable membranes has the advantage of being more "realistic", in the sense that in real reservoir systems small volumes are not restricted by semi permeable membranes. On the contrary, when using semi permeable membranes the system can be forced into a state which is "out of reach" in real systems where the application of a large pressure somewhere near a given sample volume would lead the non-wetting liquid to be forced into the surrounding porous medium, without further decreasing the water saturation in the sample volume in question.

\section{Concluding remarks}

Results from drainage experiments on transparent quasi-two-dimensional porous models have been reported. And the reduced data is used to obtain standard pressure-saturation curves.

By using different ways of driving the invasion process we can control the dynamic component of the pressure head and explain the experimentally observed dynamic effects by relating them to theoretical arguments. Indeed, by combining detailed information on the displace- 
ment structure with global measurements of pressure and saturation, we have derived a scaling relation relating pressure, saturation, system size and capillary number Ca. And by applying this pressure-saturation scaling relation, curves for a range of capillary numbers have been collapsed onto a system size- and $\mathrm{Ca}$ - independent master capillary curve. We believe that this study can have a significant impact on the understanding of the impact of dynamic effects on retention curves, and it is our hope that that the size dependence could be applied in reservoir scale modeling.

\section{Acknowledgments}

We would like to thank Henning A. Knudsen and Eirik G. Flekkøy for useful discussions and suggestions. We would also like to thank the Norwegian Research council for financial support of the project. Financial support for this research from the program REseau Alsace de Laboratoires en Ingénierie et Sciences pour l'Environnement (REALISE) is also gratefully acknowledged. R. T. and Y. M. acknowledge support from CNRS for traveling between France and Norway, under a french-norwegian PICS program.

\section{Appendix}

\section{A. Pressure adjustments}

The "gravity pump" used in the experiments consists of an open reservoir connected to the model and located a height $H$ below the model (Méheust et al., 2002). The equation of motion for the liquid is:

$$
\rho L A \phi \frac{d v}{d t}=\rho g H A-v R
$$

where $R$ is the total resistance to flow. The total resistance is dominated by resistance in the tube and valve connecting the model and the reservoir. We will therefor neglect the resistance of porous medium. Solving the velocity $v(t)$ with the boundary conditions $v(0)=0$ and $v(t \gg T)=v_{\mathrm{f}}$, one gets:

$$
v(t)=\frac{g H \rho A}{R}\left(1-e^{-\frac{t R}{\rho L A \phi}}\right)=v_{\mathrm{f}}\left(1-e^{-\frac{t}{T}}\right)
$$

where $T=L A \phi \rho / R$ is a system specific relaxation time. By using Darcy's law, and an effective pressure gradient set by the pressure drop 
exerted over the distance between the most advanced finger tip position $x=x_{\text {tip }}$ and the outlet at $x=L$ we get:

$$
v=-\frac{\kappa}{\mu_{\mathrm{w}}} \nabla P \simeq \frac{\kappa}{\mu_{\mathrm{w}}} \frac{P}{L-x_{\mathrm{tip}}}
$$

Combining Darcy's law, Eq. (17) and the definition of the capillary number (1) gives:

$$
P(t)=\frac{\mu_{\mathrm{w}}}{\kappa} v_{\mathrm{f}}\left(1-e^{-\frac{t}{T}}\right)\left(L-x_{\mathrm{tip}}\right) \approx \frac{\mathrm{Ca} \gamma}{a^{2}}\left(1-e^{-\frac{t}{T}}\right)\left(L-v_{\mathrm{tip}} t\right)
$$

where the speed of the advancing finger tip $v_{\text {tip }}$ is assumed constant. The latter equation (19) can be used to correct for the initial pressure buildup. This is done by fitting Eq. (19) to the measured $P(t)$ with $T$ and $v_{\text {tip }}$ as fitting parameters. We estimate a single characteristic $T$ by averaging the calculated $T$-values. This value is then used to correct for the initial pressure buildup, see Fig. 7 and 8.

\section{References}

Aker, E., K. J. Måløy, and A. Hansen: 2000, 'Viscous stabilization of 2D drainage displacements with trapping'. Physical Review Letters 84(20), 4589-4592.

Auradou, H., K. J. Måløy, J. Schmittbuhl, and A. Hansen: 2003, 'Drainage in a rough gouge-filled fracture'. Transport in Porous Media 50, 267-305.

Auradou, H., K. J. Måløy, J. Schmittbuhl, A. Hansen, and D. Bideau: 1999, 'Competition between correlated buoyancy and uncorrelated capillary effects during drainage'. Physical Review E 60(6), 7224-7234.

Bear, J.: 1972, Dynamics of Fluids in Porous Media. New York: American Elsevier Publishing Company.

Birovljev, A., L. Furuberg, J. Feder, T. Jøssang, K. J. Måløy, and A. Aharony: 1991, 'Gravity invasion percolation in 2 dimensions - experiment and simulation'. Physical Review Letters 67(5), 584-587.

Bohy, M., L. Dridi, G. Schäfer, and O. Razakarisoa: 2006, 'Transport of a mixture of chlorinated solvent vapors in the vadose zone of a sandy aquifer'. Vadose Zone Journal 5, 539-553.

Bourgeat, A. and M. Panfilov: 1998, 'Effective two-phase flow through highly heterogeneous porous media: Capillary nonequilibrium effects'. Computational Geosciences 2(3), 191-215.

Brooks, R. H. and A. T. Corey: 1964, Hydraulic properties of porous media. Hydrology Paper 3. Fort Collins, Colorado: Colorado State University.

Chandler, R., J. Koplik, K. Lerman, and J. F. Willemsen: 1982, 'Capillary displacement and percolation in porous media'. Journal Fluid Mechanics 119, 249-267.

Dahle, H. K., M. A. Celia, and S. M. Hassanizadeh: 2005, 'Bundle-of-tubes model for calculating dynamic effects in the capillary-pressure-saturation relationship'. Transport in Porous Media 58(1-2), 5-22. 
Dullien, F. A. L.: 1992, Porous Media Fluid Transport and Pore Structure. Sandsn Diego: Academic Press, Inc., 2nd edition.

Feder, J.: 1988, Fractals. New York: Plenum Press.

Frette, O. I., K. J. Maløy, J. Schmittbuhl, and A. Hansen: 1997, 'Immiscible displacement of viscosity-matched fluids in two-dimensional porous media'. Physical Review E 55(3), 2969-2975.

Furuberg, L., K. J. Måløy, and J. Feder: 1996, 'Intermittent behavior in slow drainage'. Physical Review E 53(1), 966-977.

Hassanizadeh, S. M., M. A. Celia, and H. K. Dahle: 2002, 'Dynamic Effect in the Capillary Pressure-Saturation Relationship and its Impacts on Unsaturated Flow'. Vadose Zone Journal 1, 38-57.

Hassanizadeh, S. M. and W. Gray: 1988, 'High-velocity flow in porous-media by Hassanizadeh and Gray - Reply'. Transport in Porous Media 3(3), 319-321.

Helmig, R.: 1997, Multiphase flow and transport processes in the subsurface. A contribution to the modeling of hydrosystems. Springer (Environmental Engineering).

Hill, S.: 1952, 'Channelling in packed columns'. Chemical Engineering Science 1, 247-253.

Kool, J. B. and J. C. Parker: 1987, 'Development and evaluation of closed form expressions for hysteretic soil hydraulic properties'. Water Resources Research 23, 105-114.

Lenhard, R. J., T. G. Johnson, and J. C. Parker: 1993, 'Experimental observations of nonaqueous-phase liquid subsurface movement'. Journal of Contaminant Hydrology 24, 79-101.

Lenormand, R.: 1989, 'Flow through porous media: limits of fractal patterns'. Proc. R. Soc. Lond. A 423, 159-168.

Lenormand, R., E. Touboul, and C. Zarcone: 1988, 'Numerical models and experiments on immiscible displacement in porous media'. Journal of Fluid Mechanics 189, 165-187.

Lenormand, R. and C. Zarcone: 1985, 'Invasion Percolation in an Etched Network: measurement of a Fractal Dimension'. Physical Review Letters 54(20), 22262229.

Lenormand, R. and C. Zarcone: 1989, 'Capillary Fingering: Percolation and Fractal Dimension'. Transport in Porous Media 4, 599-612.

Lenormand, R., C. Zarcone, and A. Sarr: 1983, 'Mechanisms of the displacement of one fluid by another in a network of capillary ducts'. Journal of Fluid Mechanics 135, 337-353.

Leverett, M. C.: 1941, 'Capillary behaviour in porous solids'. Transactions of the AIME 142, 152-169.

Løvoll, G., Y. Méheust, R. Toussaint, J. Schmittbuhl, and K. J. Måløy: 2004, 'Growth activity during fingering in a porous Hele-Shaw cell'. Phys. Rev. E $\mathbf{7 0}(2), 026301$.

Luckner, L. and M. T. H. Van Genuchten: 1989, 'A consistent set of parametric models for the two-phase flow of immiscible fluids in the subsurface'. Water Resources Research 25, 2187-2193.

Måløy, K. J., J. Feder, and T. Jøssang: 1985, 'Viscous fingering fractals in porous media'. Physical Review Letters 55, 2688-2691.

Måløy, K. J., L. Furuberg, J. Feder, and T. Jøssang: 1992, 'Dynamics of slow drainage in porous-media'. Physical Review Letters 68(14), 2161-2164.

Mandelbrot, B. B.: 1982, The Fractal Geometry of Nature. New York: W. H. Freeman. 
Méheust, Y., G. Løvoll, K. J. Måløy, and J. Schmittbuhl: 2002, 'Interface scaling in a $2 \mathrm{D}$ porous medium under combined viscous, gravity and capillary effects'. Physical Review E 66, 51603-51615.

Mualem, Y.: 1976, 'A new model for predicting the hydraulic conductivity of unsaturated porous media'. Water Resources Research 12, 513-512.

Mualem, Y.: 1984, 'A modified depend-domain theory of hysteresis'. Soil Science 137, 283-291.

Niessner, J. and S. M. Hassanizadeh: 2008, 'A model for two-phase flow in porous media including fluid-fluid interfacial area'. Water Resources Research 44(8).

Parker, J. C. and R. J. Lenhard: 1987, 'Model for hysteretic constitutive relations governing multiphase flow. 1. Saturation-pressure relations'. Water Resour. Res. 23, 2187-2196.

Parker, J. C., R. J. Lenhard, and T. Kuppusami: 1987, 'A parametric model for constitutive properties governing multiphase flow in porous media'. Water Resour. Res. 23, 618-624.

Poulavissilis, A.: 1962, 'Hysteresis of pore water, an application of concept of independent domains'. Soil Science 93, 405-412.

Saffman, P. G. and G. Taylor: 1958, 'The penetration of a fluid into a porous medium or Hele-Shaw cell containing a more viscous liquid'. Proc. Soc. London Ser. A 245, 312-329.

Sahimi, M.: 1995, Flow and Transport in Porous Media and Fractured Rock. Weinheim, Germany: VCH Verlagsgesellschaft mbH.

Schmittbuhl, J., A. Hansen, H. Auradou, and K. J. Måløy: 2000, 'Geometry and dynamics of invasion percolation with correlated buoyancy'. Physical Review E 61(4), 3985-3995.

Smiles, D., G. Vachaud, and M. Vauclin: 1971, 'A test of the uniqueness of the soil moisture characteristics during transient non-hysteretic flow of water in a rigid soil'. Soil Sci. Soc. Am. J. 35, 534-539.

Stauffer, F.: 1977, Einfluss der kapillaren Zone auf instationre Drainagevorgnge, Bericht R 13-77 (Diss ETH Nr. 5931). IHW ETH Zrich.

Stauffer, F. and W. Kinzelbach: 2001, 'Cyclic hysteretic flow in porous medium column: model, experiment, and simulations'. Journal of Hydrology 240, 264275.

Toussaint, R., G. Løvoll, Y. Meheust, K. J. Måløy, and J. Schmittbuhl: 2005, 'Influence of pore-scale disorder on viscous fingering during drainage'. Europhysics Letters 71(4), 583-589.

Van Genuchten, M. T.: 1980, 'A closed-form equation for predicting the hydraulic conductivity of unsaturated soils'. Soil Sci. Soc. Am. J. 44, 892-898.

Vedvik, A., G. Wagner, U. Oxaal, J. Feder, P. Meakin, and T. Jøssang: 1998, 'Fragmentation Transition for Invasion Percolation in Hydraulic Gradients'. Physical Review Letters 80(14), 3065-3068.

Washburn, E. W.: 1921, 'The dynamics of capillary flow'. Physical Review 17, 273-283.

Weitz, D. A., J. P. Stokes, R. C. Ball, and A. P. Kushnick: 1987, 'Dynamic capillarypressure in porous media - origin of the viscous fingering length scale'. Physical Review Letters 59(26), 2967-2970.

Wilkinson, D.: 1984, 'Percolation model of immiscible displacement in the presence of buoyancy forces'. Physical Review A 34(1), 520-531.

Wilkinson, D. and J. F. Willemsen: 1983, 'Invasion percolation: a new form of percolation theory'. J. Phys. A 16, 3365-3376. 